\title{
Hamiltonian Analysis of Plebanski Theory
}

\author{
E. Buffenoir ${ }^{a 1}$, M. Henneaux ${ }^{b 2}$, K.Noui $^{c 3}$, Ph.Roche $^{d 4}$ \\ ${ }^{a, d}$ CNRS, Laboratoire de Physique Mathématique et Théorique, Université \\ Montpellier II, 34095 Montpellier, France \\ ${ }^{b}$ Physique Théorique et Mathématique and International Solvay Institutes, \\ Université Libre de Bruxelles, B-1050 Bruxelles, Belgium. \\ ${ }^{b}$ Centro de Estudios Científicos (CECS), Valdivia, Chile. \\ ${ }^{c}$ Center for Gravitational Physics and Geometry, \\ Penn State University, State College, PA 16801, USA.
}

\begin{abstract}
We study the Hamiltonian formulation of Plebanski theory in both the Euclidean and Lorentzian cases. A careful analysis of the constraints shows that the system is non regular, i.e. the rank of the Dirac matrix is non-constant on the non-reduced phase space. We identify the gravitational and topological sectors which are regular sub-spaces of the non-reduced phase space. The theory can be restricted to the regular subspace which contains the gravitational sector. We explicitly identify first and second class constraints in this case. We compute the determinant of the Dirac matrix and the natural measure for the path integral of the Plebanski theory (restricted to the gravitational sector). This measure is the analogue of the Leutwyler-Fradkin-Vilkovisky measure of quantum gravity.
\end{abstract}

\section{Introduction}

Plebanski theory [1] is a 4-dimensional $B F$ theory with an additional field which forces the $B$ field to satisfy the simplicity constraint. By Plebanski theory we mean here the real formulation described by the real action defined below (13)

\footnotetext{
${ }^{1}$ buffenoi@lpm.univ-montp2.fr

${ }^{2}$ henneaux@ulb.ac.be

${ }^{3}$ noui@gravity.psu.edu

${ }^{4}$ roche@lpm. univ-montp2.fr
} 
and not the complex formulation whose properties have been investigated in [2, 3]. It contains, as a particular sector, 4-dimensional pure gravity and is therefore an interesting field theory. The quantum properties of this field theory are, however, largely unknown. One important line of study aims at discretizing this quantum field theory with the tools of lattice gauge field theory leading to spin-foam models. Although spin-foam models have been the subject of numerous works over these last years (see the introduction [4] and the review [5]), central issues are not understood and the technical tools needed to address these central questions need still to be developed. In particular we had in mind two pressing questions when beginning this work:

-is it possible to compute from first principle the weight of the faces, edges and vertices in the spin foam model description of Plebanski theory?

-can we see the appearance of quantum groups in Plebanski theory with cosmological constant?

The following work is a study of the Hamiltonian description of Plebanski theory. In particular we want to address the following problems:

-computation of the Liouville measure in the path integral expressed in term of the original variables of Plebanski theory. This could be a first step for understanding how to fix the measure of spin foam models.

-computation of the Dirac bracket of all the fields once all second class constraints have been taken into account. In particular one could have expected to find some brackets of quadratic type which are the signature of quantum groups. Unfortunately the Dirac brackets of the variables are complicated and no hint of quantum groups have appeared yet using this method.

Our paper is organized as follows. In the next section (section II), we briefly recall the Plebanski description of gravity as a constrained $B F$ theory. We then give the Hamiltonian analysis of the theory (primary and secondary constraints, first and second class constraints)(section III). We finally derive the path integral measure (section IV).

\section{Gravity as a Constrained Topological Field Theory}

This section is devoted to briefly reviewing the classical Lagrangian analysis of $B F$ and Plebanski theories, and to presenting the notations that we are using throughout the work. Let $\mathcal{M}$ be a four dimensional oriented smooth manifold $\mathcal{M}$ and $\Omega^{1}(\mathcal{M})$ the space of one-forms defined on the manifold $\mathcal{M}$. A local coordinate system will be denoted by $x^{\mu}$, with $\mu \in\{0,1,2,3\}$. 


\section{II.1. BF action with cosmological term}

\section{II.1.1. Dynamics}

For any Lie group $G$ one can define a $B F$ theory which is a topological quantum field theory [6, 7, 8]. In the sequel we will only be interested in the case where the group $G=S O(\eta)$ with $\eta=\operatorname{diag}\left(\sigma^{2}, 1,1,1\right)$ and $\sigma=1$ (resp. $\sigma=i$ ). We will coin the name Riemannian (resp. Lorentzian) BF-theory in these cases. As usual, we will denote $\mathfrak{g}=s o(\eta)$ the Lie algebra of the group whose basic properties are recalled in the appendix A. By convention, given two $\mathfrak{g}$-valued one forms $\alpha=\alpha_{\mu} d x^{\mu}$ and $\beta=\beta_{\mu} d x^{\mu}$, their commutator is defined to be the $\mathfrak{g}$-valued twoform $[\alpha, \beta]=\left[\alpha_{\mu}, \beta_{\nu}\right] d x^{\mu} \wedge d x^{\nu}$ whereas the exterior product of these forms is given by $\alpha \wedge \beta=\frac{1}{2}[\alpha, \beta]$. We will also make an extensive use of the symmetrization notation: $\gamma_{(\mu \nu)}=\frac{1}{2}\left(\gamma_{\mu \nu}+\gamma_{\nu \mu}\right)$ for any matrix $\gamma$.

The action defining $B F$-theory with a cosmological constant $\Lambda$ is a functional $S_{B F}[A, B]$ of a $\mathfrak{g}$-valued two-form $B=\frac{1}{2} B_{\mu \nu} d x^{\mu} \wedge d x^{\nu}$, the $B$-field, and a $\mathfrak{g}$ connection $A=A_{\mu} d x^{\mu}$ given by:

$$
S_{B F}[A, B]=\frac{1}{2} \int_{\mathcal{M}} d^{4} x \epsilon^{\mu \nu \rho \sigma}\left(<B_{\mu \nu}, F_{\rho \sigma}>+\frac{\Lambda}{2} \prec B_{\mu \nu}, B_{\rho \sigma} \succ\right)
$$

where $F=d A+A \wedge A=\frac{1}{2} F_{\mu \nu} d x^{\mu} \wedge d x^{\nu}$ is the curvature of the connection $A$, $\epsilon^{\mu \nu \rho \sigma}$ is the Levi-Civita symbol and the two independent Killing forms on the Lie algebra, $\langle\cdot, \cdot\rangle$ and $\prec \cdot, \cdot \succ$, are precisely defined in the appendix A (see (82)). As explained in the next section, the choice of the second Killing form in the cosmological term is made so that the full Plebanski action, obtained by adding the so-called simplicity constraints to (1I), is equivalent (in the appropriate sector) to ordinary gravity.

Although this theory is well-known to be a topological field theory (see [4] for example), it still contains many unsettled questions such as the evaluation of the expectation values of observables (see [8] as well as more recent articles on this subject.)

The equations of motion of $B F$ action with respect to the fields $B$ and $A$ are respectively given by:

$$
\iota(F)+\Lambda B=0 \text { and } D B \equiv d B+[A, B]=0 .
$$

In these equations, $\iota$ denotes the Hodge map defined in the appendix A. The situation is therefore quite different in the cases $\Lambda=0$ and $\Lambda \neq 0$.

The $B$-field is locally covariantly constant but in the case where $\Lambda \neq 0$, is completely determined once the connection $A$ is given. Furthermore, in that case, the second equation (22) is an immediate consequence of the first one and the

Bianchi identity. Any solution of the equations of motion is therefore given by a 
couple $(A, B)$ with $A$ any connection and $B$ given by $B=-\Lambda^{-1} \iota(F)$. When the cosmological constant is zero, any solution of the equations of motion is given by a couple $(A, B)$ with $A$ any flat connection and $B$ covariantly constant. Moreover, as the connection $A$ is flat, the $B$-field can be locally written as $B=D \omega$ for some $\mathfrak{g}$-valued one-form $\omega$.

\section{II.1.2 Gauge symmetries}

We now discuss the symmetries of the $B F$-action. First of all, we have the group of gauge transformations $\mathcal{G}=C^{\infty}(\mathcal{M}, G)$ whose action on the fields is given by:

$$
\forall g \in \mathcal{G}, A^{g}=g^{-1} A g+g^{-1} d g \quad \text { and } \quad B^{g}=g^{-1} B g .
$$

The invariance of the action (11) under the group of gauge transformations is an immediate consequence of the invariance of the Killing forms (82). Besides these familiar gauge transformations, $B F$-theory admits another set of symmetries $\mathcal{T}=\Omega^{1}(\mathcal{M}) \otimes \mathfrak{g}$ whose action on the fields depends explicitly on the cosmological constant as follows:

$$
\forall \omega \in \mathcal{T}, \quad A^{\omega}=A-2 \Lambda \iota(\omega) \text { and } B^{\omega}=B+D \omega-2 \Lambda \iota([\omega, \omega])
$$

The proof of the $B F$-action invariance under these transformations is a straightforward computation and uses, as a central tool, the property (83) and the Killing forms invariance.

The symmetry group $\mathcal{S}$ of the action (11) is obtaining by combining (3) and (41), i.e., is the semidirect product $\mathcal{S}=\mathcal{G} \ltimes \mathcal{T}$ where the action on the fields is given by $(A, B) \triangleleft(g, \omega)=\left(\left(A^{g}\right)^{\omega},\left(B^{g}\right)^{\omega}\right)$. The composition law between two elements $\left(g_{1}, \omega_{1}\right)$ and $\left(g_{2}, \omega_{2}\right)$ of $\mathcal{S}$ is given by:

$$
\left(g_{1}, \omega_{1}\right) \circ\left(g_{2}, \omega_{2}\right)=\left(g_{1} g_{2}, \omega_{2}+g_{2}^{-1} \omega_{1} g_{2}\right) .
$$

At this point, it is interesting to note that, in contrast to the three-dimensional case [9], the symmetry group structure (5) is the same for any value of $\Lambda$. The cosmological constant appears only in the expression of the action of the normal subgroup $\mathcal{T}$ on the fields (41).

The infinitesimal expression of the symmetries easily follows from (3, 4) and reads :

$$
\delta_{(u, \tau)} A=D u-2 \Lambda \iota(\tau) \quad \text { and } \quad \delta_{(u, \tau)} B=B+[u, B]+D \tau
$$

where $u \in C^{\infty}(\mathcal{M}, \mathfrak{g})$ and $\tau \in \Omega^{1}(\mathcal{M}) \otimes \mathfrak{g}$.

Moreover, being given by the integral of a 4 -form, the $B F$-action is also invariant under the group of diffeomorphisms of $\mathcal{M}$. In the case of Riemannian or 
Lorentzian BF theory, there is an interplay between the action of diffeomorphisms and the action of the symmetry group $\mathcal{S}$. To see that this is indeed the case, let us compute the Lie derivative $\mathcal{L}_{\xi}$ of the fields $A$ and $B$ along a vector field $\xi=\xi^{\mu} \partial_{\mu}$. An easy calculation shows that these Lie derivatives are related to the infinitesimal symmetries (6) as follows:

$$
\begin{aligned}
\left(\mathcal{L}_{\xi} A\right)_{\mu}^{(\varepsilon) a} & =\left(\delta_{(u, \tau)} A\right)_{\mu}^{(\varepsilon) a}+\xi^{\rho} \frac{\delta S_{B F}}{\delta B_{\rho \mu}^{(\varepsilon) a}} \\
\left(\mathcal{L}_{\xi} B\right)_{\mu \nu}^{(\varepsilon) a} & =\left(\delta_{(u, \tau)} B\right)_{\mu \nu}^{(\varepsilon) a}-\epsilon_{\mu \nu \rho \sigma} \xi^{\rho} \frac{\delta S_{B F}}{\delta A_{\sigma}^{(\varepsilon) a}}
\end{aligned}
$$

where $u=\xi^{\mu} A_{\mu}$ and $\tau=\xi^{\rho} B_{\rho \mu} d x^{\mu}$. As a result, if the fields $A$ and $B$ satisfy the equations of motion (2), their transformations under diffeomorphisms can be expressed only in terms of the symmetries (6), which form a complete set in the sense of [10].

It follows from the form of the gauge transformations that all the solutions are locally gauge-related on-shell. When $\Lambda \neq 0$, two solutions of the equations of motion are related by a symmetry $\omega$. When $\Lambda=0$, two solutions of the equation of motion are such that the respective connections are flat, so they are locally pure gauge and therefore are related by a gauge transformation. The respective $B$ fields are covariantly constant, therefore locally there exists $\omega$ which relates the two.

\section{II.1.3. Hamiltonian analysis}

Let us end up this brief review on $B F$ theory by recalling basic facts [6, 4] about the Hamiltonian analysis (see [11] for a recent complete treatment).

For this purpose, we assume that spacetime has the structure $\mathcal{M}=\mathbb{R} \times \Sigma$ where the real line $\mathbb{R}$ represents time and $\Sigma$ is an oriented smooth 3-manifold representing space. We will use a chart $x^{\mu}$ adapted to this foliation: $x^{0}$ is a coordinate on $\mathbb{R}$ whereas $x^{i}, i=1,2,3$ are coordinates on $\Sigma$. As usual we denote $\epsilon^{i j k}=\epsilon^{0 i j k}$.

The action (11) shows that the spatial components of the connection $A_{i}^{(\varepsilon) a}$ and $\epsilon^{i j k} B_{j k}^{(\varepsilon) b} \eta_{a b}=\Pi_{(\varepsilon) a}^{i}$ are canonically conjugate fields, i.e.

$$
\left\{A_{i}^{(\varepsilon) a}(x), \Pi_{\left(\varepsilon^{\prime}\right) b}^{j}(y)\right\}=\delta_{a}^{b} \delta_{\varepsilon, \varepsilon^{\prime}} \delta_{i}^{j} \delta(x, y)
$$

Moreover, a straightforward calculation shows that the canonical Hamiltonian $H_{B F}[A, B]$ reads:

$$
H_{B F}[A, B]=-\int_{\Sigma} d^{3} x\left(\epsilon^{i j k}<B_{0 i}, F_{j k}>+<A_{0}, D_{i} \Pi^{i}>+\Lambda \prec B_{0 i}, \Pi^{i} \succ\right) .
$$


The components $A_{0}^{(\varepsilon) a}$ of the connection and the components $B_{0 i}^{(\varepsilon) a}$ of the $B$-field appear to be non-dynamical variables: they are Lagrange multipliers which impose the following primary constraints:

$$
\begin{aligned}
& \epsilon^{i j k} \iota\left(F_{j k}\right)+\Lambda \Pi^{i}=0 \\
& D_{i} \Pi^{i}=0 .
\end{aligned}
$$

These constraints are obviously contained in the Lagrangian equations (2), are first class and generate the infinitesimal symmetries. Transformation laws of the dynamical variables $\left(A_{i}^{(\varepsilon) a}, \Pi_{(\varepsilon) a}^{i}\right)$ under these symmetries are given by (6) with parameters $u$ and $\tau$ such that $\tau_{0}=0$.

\section{II.2. Plebanski action}

Plebanski action [1] is a constrained Riemannian or Lorentzian BF theory, in which the simplicity constraint on the $B$ field written explicitly in (14) below is dynamically enforced through the introduction of Lagrange multipliers.

The Plebanski action is a functional $S_{P l}[A, B, \varphi]$ of the $B$-field, the connection $A$ and Lagrange multipliers $\varphi$. Actually, there exists two different but "equivalent" ${ }^{5}$ formulations for this action [12] depending on the choice of the Lagrange multipliers type: $\varphi$ can be a density tensor field with space-time indices only or a field with Lie-algebra indices (internal) only. The latter formulation is more customary in the literature on spin foams but we will use the former which turns out to be more convenient for the achievement of an Hamiltonian analysis. The $S O(\eta)$ Plebanski action is given by:

$$
S^{P l}[A, B, \varphi]=S_{B F}[A, B]+\frac{1}{4} \int_{\mathcal{M}} d^{4} x \varphi^{\mu \nu \rho \sigma} \prec B_{\mu \nu}, B_{\rho \sigma} \succ .
$$

In this expression, $\varphi^{\mu \nu \rho \sigma}=\varphi^{[\mu \nu][\rho \sigma]}$ is a density tensor, symmetric under the exchange of the pair $[\mu \nu]$ with $[\rho \sigma]$ and satisfying the tracelessness condition: $\epsilon_{\mu \nu \rho \sigma} \varphi^{\mu \nu \rho \sigma}=0$. Therefore it contains 20 independent components. Varying the action (13) with respect to $\varphi$ imposes the following 20 algebraic equations on the 36 components of the $B$-field:

$$
\prec B_{\mu \nu}, B_{\rho \sigma} \succ=\frac{\mathcal{V}}{4 !} \epsilon_{\mu \nu \rho \sigma}
$$

where $\mathcal{V}=\epsilon^{\mu \nu \rho \sigma} \prec \mathcal{B}_{\mu \nu}, \mathcal{B}_{\rho \sigma} \succ$. In the sequel, $\mathcal{V}$ will be called the four-dimensional volume. When $\mathcal{V}$ is not vanishing it is said that the non degeneracy condition is satisfied. The equations (14) are known as "simplicity constraints" and their

\footnotetext{
${ }^{5}$ To be more precise, the two formulations are shown to be equivalent on shell when the non degeneracy condition is satisfied.
} 
properties and solutions have been studied and classified when the non degeneracy condition is satisfied [13, 14, 12. In particular, it was shown that they are equivalent to the following system of equations:

$$
\epsilon^{\mu \nu \rho \sigma} B_{\mu \nu}^{I J} B_{\rho \sigma}^{K L}=\frac{\mathcal{V}}{4 !} \epsilon^{I J K L} .
$$

Moreover, simplicity conditions and the non degeneracy conditions are simultaneously fullfilled if and only if there exists a non degenerate cotetrad field $e^{I}=e_{\mu}^{I} d x^{\mu}$ such that the $B$-field can be written in one of the following forms:

$$
\begin{aligned}
\text { topological sector }(\mathrm{I} \pm) & : B^{I J}= \pm e^{I} \wedge e^{J} \\
\text { gravitational sector }(\mathrm{II} \pm) & : B^{I J}= \pm \frac{1}{2} \epsilon^{I J}{ }_{K L} e^{K} \wedge e^{L}
\end{aligned}
$$

We now come to the relation between Plebanski theory and Palatini formulation of general relativity. If one substitutes the solutions of the sector $(I I \pm)$ (17) into the Plebanski action, one obtains $\pm S_{ \pm \Lambda}^{P a}[A, e]$, where $S_{\Lambda}^{P a}[A, e]$ is the Palatini action of general relativity with cosmological constant $\Lambda$ :

$$
S_{\Lambda}^{P a}[A, e]=\frac{1}{4} \int_{\mathcal{M}} d^{4} x \epsilon^{\mu \nu \rho \sigma} \epsilon_{I J K L} e_{\mu}^{I} e_{\nu}^{J}\left(F_{\rho \sigma}^{K L}+\frac{\Lambda}{4} e_{\rho}^{K} e_{\sigma}^{L}\right) .
$$

This result gives an a posteriori justification of the choice for the Killing form defining the cosmological term of the $B F$-action (11). The topological sector however does not have any physical interpretation.

Note that when the non degeneracy condition is fulfilled the different set of solutions of the simplicity conditions are disjoint. To see that this is the case, the Urbantke metrics $g_{\mu \nu}^{(\varepsilon)}, \varepsilon \in\{+1,-1\}$, defined as [15]:

$$
g_{\mu \nu}^{(\varepsilon)}=-\frac{2}{3 \mathcal{V}} \epsilon_{a b c} \epsilon^{\alpha \beta \gamma \delta} B_{\mu \alpha}^{(\varepsilon) a} B_{\beta \gamma}^{(\varepsilon) b} B_{\delta \nu}^{(\varepsilon) c} .
$$

are a useful tool. If the $B$-field satisfies the simplicity conditions, their expressions simplify drastically and are given in term of the cotetrad $e_{\mu}^{I}$ by the formulae:

$$
\begin{aligned}
\text { topological sector }(I \pm) & : g_{\mu \nu}^{(\varepsilon)}= \pm \varepsilon \eta_{I J} e_{\mu}^{I} e_{\nu}^{J} \\
\text { gravitational sector }(I I \pm) & : g_{\mu \nu}^{(\varepsilon)}= \pm \eta_{I J} e_{\mu}^{I} e_{\nu}^{J}
\end{aligned}
$$

As a result, a non-degenerate $B$-field solution of the simplicity constraints cannot belong to both topological and gravitational sectors. Moreover, given a $B$-field in the gravitational or topological sector, the signature of its associated Urbantke metrics completely fixes its belonging to the $( \pm)$ sectors. The four sectors are therefore disjoint. 


\section{Hamiltonian analysis of Plebanski theory}

We now study $S O(\eta)$ Plebanski theory in the Hamiltonian framework. For this purpose, we will assume that $\mathcal{M}=\mathbb{R} \times \Sigma$ where $\Sigma$ is a smooth, oriented threemanifold (without boundary) which will play the role of space.

The first step consists in deriving the Hamiltonian and the constraints. One must then split the constraints into first class constraints and second class constraints. A notable feature of the Plebanski action is that it does not define a regular Hamiltonian system in the sense that the rank of the Dirac matrix depends on which type of sectors the $B$-field belongs to.

We provide a set of holonomic constraints which replace the simplicity constraints and eliminate the topological sector. When the $B$-field is non holonomically enforced to belong to the gravitational sector $I I+$ we can compute the Dirac

matrix determinant in closed form. This will be used in section IV to formulate a path integral quantization of Plebanski theory in this sector.

\section{III.1. Constraint analysis}

The Plebanski action (13) can be written, after an integration by parts, as:

$$
\begin{aligned}
& S_{P l}=\int d t \int_{\Sigma} d^{3} x\left(\epsilon^{m n p}<B_{m n}, \partial_{0} A_{p}>+\epsilon^{m n p}<B_{0 p}, F_{m n}>+\right. \\
& <A_{0}, D_{p} B_{m n}>\epsilon^{m n p}+\Lambda \prec B_{0 p}, B_{m n} \succ \epsilon^{m n p}+ \\
& \left.\varphi^{0 m 0 n} \prec B_{0 m}, B_{0 n} \succ+\varphi^{0 m n p} \prec B_{0 m}, B_{n p} \succ+\frac{1}{4} \varphi^{m n r s} \prec B_{m n}, B_{r s} \succ\right) .
\end{aligned}
$$

From this expression we see that the fields $A_{0}, B_{0 m}, \varphi$ are non dynamical since their time derivatives do not occur in the Lagrangian. However the fact that the $B_{0 m}$ 's appear quadratically prevents us from treating them as Lagrange multipliers. To handle this problem, we decided to add to the Lagrangian the term

$$
<P^{i}, \partial_{0} B_{0 i}>-<\mu_{i}, P^{i}>
$$

where $P^{i}$ and $\mu_{i}$ are new independent variables. This does not change the dynamics since the new fields are auxiliary fields, the elimination of which yields back the original action (by varying with respect to $\mu_{i}$ and $P^{i}$, one gets $P^{i}=0$ and $\left.\partial_{0} B_{0 i}-\mu_{i}=0\right)$. This modified action with the new fields is our starting point.

\section{III.1.1. Conjugate momenta and primary constraints}

With the new variables, the Plebanski action is in first order form:

$S_{P l}^{(1)}\left[A_{i}, \Pi_{i}, P_{i}, B_{0 i}, A_{0}, \mu_{i}, \varphi\right]=\int d t \int_{\Sigma} d^{3} x\left(<\Pi^{i}, \partial_{0} A_{i}>+<P^{i}, \partial_{0} B_{0 i}>-H\right)(23)$ 
where we have made the change of variables

$$
\Pi^{i}=\epsilon^{m n i} B_{m n}
$$

and where the Hamiltonian $H$ reads:

$$
\begin{aligned}
& -H=\epsilon^{m n p}<B_{0 p}, F_{m n}>+<A_{0}, D_{p} \Pi^{p}>+\Lambda \prec B_{0 p}, \Pi^{p} \succ+ \\
& +\varphi^{0 m 0 n} \prec B_{0 m}, B_{0 n} \succ+\frac{1}{2} \varphi^{0 m n p} \prec B_{0 m}, \Pi^{r} \succ \epsilon_{n p r}+ \\
& +\frac{1}{16} \varphi^{m n r s} \epsilon_{m n i} \epsilon_{r s j} \prec \Pi^{i}, \Pi^{j} \succ-<\mu_{i}, P^{i}>.
\end{aligned}
$$

We will denote $\mathcal{V}=\prec B_{0 j}, \Pi^{j} \succ$ and assume that the non degeneracy condition holds i.e $\mathcal{V} \neq 0$. We conclude immediately that the only dynamical fields are the spatial components of the connection $A_{i}$, their conjugate momenta $\Pi^{i}$ as well as the $B_{0 i}$ and their momenta $P^{i}$. The other fields $A_{0}, \mu_{i}$ and $\varphi$ do not appear with time derivatives. Moreover, the action (23) is linear in them. They can, therefore, be considered as Lagrange multipliers. Thus, we do not associate conjugate momenta to these variables in order not to complicate unecessarily the canonical analysis and we start with the phase space of the $A_{i}$ 's, the $\Pi^{i}$ 's, the $B_{0 i}$ 's and the $P^{i}$ 's with following non-zero Poisson brackets :

$$
\begin{aligned}
& \left\{A_{i}^{(\varepsilon) a}(x), \Pi_{\left(\varepsilon^{\prime}\right) b}^{j}(y)\right\}=\delta_{b}^{a} \delta_{\varepsilon, \varepsilon^{\prime}} \delta_{j}^{i} \delta(x, y) \\
& \left\{B_{0 i}^{(\varepsilon) a}(x), P_{\left(\varepsilon^{\prime}\right) b}^{i}(y)\right\}=\delta_{b}^{a} \delta_{\varepsilon, \varepsilon^{\prime}} \delta_{j}^{i} \delta(x, y) .
\end{aligned}
$$

The "primary constraints" are given by

$$
\begin{aligned}
& P_{(\varepsilon) a}^{i} \approx 0 \\
& \Gamma \equiv D_{p} \Pi^{p} \approx 0 \\
& \Phi(B, B)_{i j} \equiv \prec B_{0 i}, B_{0 j} \succ \approx 0, \\
& \Phi(B, \Pi)_{j}^{i} \equiv \prec B_{0 j}, \Pi^{i} \succ-\frac{1}{3} \delta_{j}^{i} \mathcal{V} \approx 0, \\
& \Phi(\Pi, \Pi)^{i j} \equiv \prec \Pi^{i}, \Pi^{j} \succ \approx 0 .
\end{aligned}
$$

The constraint (28) is obtained by varying with respect to $\mu_{i}$, constraint (29) is obtained by varying with respect to $A_{0}$ and the simplicity constraints (30 3132) are obtained by varying with respect to $\varphi$. The time evolution of any phase space function $f$ is given by $\dot{f}=\{f, H\}$.

\section{III.1.1. Secondary constraints}

We now study the secondary constraints arising from the conservation of the primary ones. 
Conservation of the constraints (28) under time evolution implies the following conditions:

$$
\dot{P}^{i}=-\epsilon^{i j k} F_{j k}+\sigma \Lambda \iota\left(\Pi^{i}\right)+2 \sigma \varphi^{0 i 0 j} \iota\left(B_{0 j}\right)+\sigma \varphi^{0 i j k} \epsilon_{j k l} \iota\left(\Pi^{l}\right) \approx 0 .
$$

These 18 equations involve the 6 components $\varphi^{0 i 0 j}$ and the 8 components $\varphi^{0 i j k}$. Due to the invertibility of $\mathcal{V}$ and the expressions of the primary simplicity constraints, 14 of these equations completely fix the value of $\varphi^{0 i 0 j}, \varphi^{0 i j k}$ whereas the 4 remaining ones are secondary constraints given by:

$$
\begin{aligned}
& C_{0} \equiv<\dot{P}^{i}, B_{0 i}>=\epsilon^{i j k}<B_{0 i}, F_{j k}>+\Lambda \prec \Pi^{i}, B_{0 i} \succ \approx 0, \\
& C_{i} \equiv \epsilon_{i j k}<\dot{P}^{j}, \Pi^{k}>=<F_{i j}, \Pi^{j}>\approx 0 .
\end{aligned}
$$

Conservation of the constraint (29) does not generate any new secondary constraint. To see this it is useful (and permissible) to redefine the constraint $\Gamma$ as follows:

$$
\Upsilon=\Gamma-\left[P^{i}, B_{0 i}\right] \approx 0
$$

The constraint $\Upsilon$ generates infinitesimal gauge transformations of type $\mathcal{G}$ on all the dynamical variables. As a result, the constraints $\Upsilon$ are conserved under time evolution because $H$ is invariant under these transformations.

Before studying the conservation of the simplicity constraints we prove some relations implied by the non degeneracy condition. We denote by $K, L$ the $6 \times 6$ matrices defined by

$$
K_{i}^{(\varepsilon) a}=B_{0 i}^{(\varepsilon) a}, K_{3+i}^{(\varepsilon) a}=\Pi_{(\varepsilon) a}^{i}, L_{(\varepsilon) a}^{3+i}=\frac{3 \epsilon}{\sigma \mathcal{V}} B_{0 i}^{(\varepsilon) a}, L_{(\varepsilon) a}^{i}=\frac{3 \epsilon}{\sigma \mathcal{V}} \Pi_{(\varepsilon) a}^{i} i=1, \ldots, 3 .
$$

The non degeneracy condition and the simplicity constraints imply that $L$ is the weak left inverse of $K$. This implies that $L$ is also the right weak inverse of $K$ which reads:

$$
\sum_{k} \Pi_{(\varepsilon) a}^{k} B_{0 k}^{\left(\varepsilon^{\prime}\right) b}+\Pi_{\left(\varepsilon^{\prime}\right) b}^{k} B_{0 k}^{(\varepsilon) a} \approx \frac{\varepsilon}{3 \sigma} \mathcal{V} \delta_{a}^{b} \delta_{\varepsilon, \varepsilon^{\prime}}
$$

First, we consider the set of constraints (30 31) whose time derivative is given by:

$$
\begin{aligned}
\dot{\Phi}(B, B)_{i j}=2 \prec \mu_{(i}, B_{0 j} \succ \approx 0, \\
\dot{\Phi}(B, \Pi)_{j}^{i}=\prec \mu_{i}, \Pi^{j} \succ+2 \epsilon^{i m n} \prec B_{0 j}, D_{m} B_{0 n} \succ+<A_{0},\left[B_{0 j}, \Pi^{i}\right]> \\
-\frac{1}{3} \delta_{j}^{i}\left(\prec \mu_{k}, \Pi^{k} \succ+2 \epsilon^{k m n} \prec B_{0 k}, D_{m} B_{0 n} \succ+<A_{0},\left[B_{0 k}, \Pi^{k}\right]>\right) \approx 0 .
\end{aligned}
$$

These 14 equations fix 14 independant linear combinations among the 18 multipliers $\mu_{i}^{(\varepsilon) a}$ and imply no further constraint on the dynamical variables. Indeed, let us introduce the variables $\nu_{i}^{m}$ with $\mu_{i}^{(\varepsilon) a}=\sum_{m=1}^{6} \nu_{i}^{m} K_{m}^{(\varepsilon) a}$. The set of constraints 
(3940) fix the combinations $\nu_{j}^{3+i}+\nu_{i}^{3+j}$ and $\nu_{j}^{i}-\frac{1}{3} \delta_{j}^{i} \sum_{k=1}^{3} \nu_{k}^{k}$ where $i, j \in\{1,2,3\}$. From this analysis, we can reorganize the 18 constraints $P^{i} \approx 0$ in two subsets of constraints:

$$
\begin{aligned}
& \kappa_{0}=<P^{i}, B_{0 i}>\approx 0 \\
& \kappa_{i}=\epsilon_{i j k}<P^{j}, \Pi^{k}>\approx 0 \\
& \Phi(B, P)_{j}^{i}=<P^{i}, B_{0 j}>-\frac{1}{3} \delta_{j}^{i}<P^{k}, B_{0 k}>\approx 0 \\
& \Phi(P, \Pi)^{i j}=<P^{(i}, \Pi^{j)}>\approx 0 .
\end{aligned}
$$

We will show later that $\kappa_{0}, \kappa_{i}$ are the 4 first class constraints corresponding to the previous four Lagrange multipliers among the $\mu$ 's that remain arbitrary.

The study of the conservation of the remaining simplicity constraints (32) introduces, by contrast, new constraints in the theory. Indeed, vanishing of the time derivatives of these constraints imposes the following conditions:

$$
-\frac{1}{4} \dot{\Phi}(\Pi, \Pi)^{i j} \equiv T^{i j}=\epsilon^{m n(i} \prec D_{m} \Pi^{j)}, B_{0 n} \succ \approx 0
$$

This closes the analysis of the secondary constraints.

\section{III.1.3. Conservation of secondary constraints}

In order to complete the Dirac algorithm we have to study the conservation of these secondary constraints. A direct computation shows that time derivative of the constraints (45) can be expressed as:

$$
\begin{aligned}
& \dot{T}^{i j}=-2 \epsilon^{u v(i} \epsilon^{m j) p} \prec D_{u} D_{m} B_{0 p}, B_{0 v} \succ+\epsilon^{m n(i} \prec\left[A_{0}, B_{0 n}\right], D_{m} \Pi^{j)} \succ+ \\
& +\sigma^{2} \Lambda \epsilon^{m n(i}<\Pi^{j)},\left[B_{0 m}, B_{0 n}\right]>+\epsilon^{m n(i} \prec D_{m} \Pi^{j)}, \mu_{n} \succ+\sigma^{2} \varphi^{0 k l(i}<\Pi^{j)},\left[B_{0 k}, B_{0 l}\right]> \\
& +\frac{1}{4} \sigma^{2} \varphi^{m n r s} \epsilon_{m n k} \epsilon_{r s l}<\Pi^{(k} \epsilon^{l) v(i},\left[\Pi^{j)}, B_{0 v}\right]>\approx 0 .
\end{aligned}
$$

As a result, conservation of the constraints $T^{i j}$ is a system of equations which will eventually fix some of the yet unfixed Lagrange multipliers $\varphi^{k l m n}$. This point depends crucially on invertibility properties of the following tensor:

$$
G^{u v i j}=\sigma^{2}<\Pi^{(v} \epsilon^{u) r(i},\left[\Pi^{j)}, B_{0 r}\right]>.
$$

We now show that $G^{u v i j}$ can be rewritten in terms of the Urbantke metrics. It follows that its properties depend on the sector under consideration.

Lemma 1 The tensor $G^{u v i j}$ satisfies the identity:

$$
G^{u v i j} \approx \frac{\sigma^{2}}{4} \mathcal{V}\left(\epsilon^{k i(u} \epsilon^{v) l j}+\epsilon^{k j(u} \epsilon^{v) l i}\right)\left(g_{k l}^{(+)}+g_{k l}^{(-)}\right) .
$$


This implies that:

-in the topological sector (I) the matrix $G$ identically vanishes.

-in the non topological sector (II) the matrix $G$ is invertible and its determinant reads:

$$
\operatorname{det}(G) \approx-\frac{1}{4} \mathcal{V}^{6}\left(\operatorname{det}\left(g_{k l}\right)\right)^{2}
$$

Proof: Using the formula defining the Urbantke metrics, and the formula expressing $B_{i j}$ in term of $\Pi$, we get:

$$
g_{i j}^{(\epsilon)} \approx \frac{1}{\mathcal{V}} \epsilon_{a b c} \epsilon_{m(i r} B_{0 j)}^{(\varepsilon) a} \Pi_{(\varepsilon) b}^{m} \Pi_{(\varepsilon) c}^{r}
$$

Using this result we can now compute the right handside of (47), which is equal to $G^{\text {uvij }}$.

We have previously seen that the topological phase (I) is characterized by the condition $g_{\mu \nu}^{(+)}+g_{\mu \nu}^{(-)}=0$. This implies that $G$ is zero in this sector. In the non-topological sector we have $G^{u v i j} \approx \frac{\sigma^{2}}{2} \mathcal{V}\left(\epsilon^{k i(u} \epsilon^{v) l j}+\epsilon^{k j(u} \epsilon^{v) l i}\right) g_{k l}$. From this expression one easily computes the determinant $\operatorname{det}(G)$ :

$$
\operatorname{det}(G) \approx c \mathcal{V}^{6}\left(\operatorname{det}\left(g_{k l}\right)\right)^{2}
$$

where the constant $c$ is evaluated to be $c=-\frac{1}{4}$ (go to a frame where $g_{k l}=\delta_{k l}$ ).

In the sequel, we will denote by $V_{s}$ the spatial volume defined by $V_{s}^{2}=$ $\left|\operatorname{det}\left(g_{k l}\right)\right|$, so we have $\operatorname{det}(G) \approx c \mathcal{V}^{6} V_{s}^{4}$.

Because the matrix $G$ is invertible in the non topological sector, the equations $\dot{T}^{i j}=0$ fix the value of the 6 remaining Lagrange multipliers $\varphi^{k l m n}$ and do not impose any new constraints. By contrast, in the topological sector, these equations do not fix these Lagrange multipliers anymore but give tertiary constraints. The Plebanski theory turns out to define a non-regular Hamiltonian system, i.e. its constraint analysis is not uniform on the non-reduced phase space. In the topological sector, we could continue the Dirac algorithm and we would observe that the set of first class constraints is larger than in the non topological sector. The gauge symmetries generated by this set of first class constraints would absorb all the local dynamical degrees of freedom. This justifies the term "topological" for this sector. In the sequel, we will exclusively concentrate on the non topological sector.

It now remains to understand the conservation in time of the constraints $C_{0}, C_{i}$. By anticipating the discussion concerning separation of the constraints between first class and second class, we will modify the constraints $C_{0}, C_{i}$ by adding to them linear combination of constraints in such a way that the resulting constraints, denoted $C_{0}^{\prime}, C_{i}^{\prime}$, are first class. 
This is possible: let us define

$$
\begin{aligned}
C_{i}^{\prime} & =C_{i}+<P^{j}, D_{i} B_{0 j}>-<A_{i}, \Upsilon>-\partial_{j}<P^{j}, B_{0 i}> \\
& =<\partial_{i} A_{j}, \Pi^{j}>-\partial_{j}<A_{i}, \Pi^{j}>+<P^{j}, \partial_{i} B_{0 j}>-\partial_{j}<P^{j}, B_{0 i}>.
\end{aligned}
$$

We have verified by direct computation that $C_{i}^{\prime}$ are indeed first class constraints (the only non trivial computations are the weak Poisson commuting property with respect to $T^{i j}$ and $C_{0}$ ). Consequently, they are conserved under time evolution. As to the constraint $C_{0}^{\prime}$, we define it as being the total Hamiltonian with the values of the Lagrange multipliers fixed by the Dirac analysis: this is precisely solving the condition that $C_{0}^{\prime}$ is first class with $C_{0}^{\prime}-C_{0}$ a linear combination of constraints.

\section{III.2 First and Second Class constraints}

\section{III.2.1. Separation of constraints}

Once all the constraints have been determined, one must split them into first class and second class constraints. To achieve this (in the non topological sector), we reorganize the set of all the constraints as follows:

$$
\begin{aligned}
& \kappa_{0}=<P^{i}, B_{0 i}>\approx 0 \\
& \kappa_{i}=\epsilon_{i j k}<P^{j}, \Pi^{k}>\approx 0, \\
& \Phi(B, P)_{j}^{i}=<P^{i}, B_{0 j}>-\frac{1}{3} \delta_{j}^{i}<P^{k}, B_{0 k}>\approx 0 \\
& \Phi(P, \Pi)^{i j}=<P^{(i}, \Pi^{j)}>\approx 0 \\
& \Upsilon=D_{p} \Pi^{p}-\left[P^{p}, B_{0 p}\right] \approx 0 \\
& \Phi(B, B)_{i j}=\prec B_{0 i}, B_{0 j} \succ \approx 0 \\
& \Phi(B, \Pi)_{j}^{i}=\prec B_{0 j}, \Pi^{i} \succ-\frac{1}{3} \delta_{j}^{i} \mathcal{V} \approx 0 \\
& \Phi(\Pi, \Pi)^{i j}=\prec \Pi^{i}, \Pi^{j} \succ \approx 0 \\
& T^{i j}=\epsilon^{m n(i} \prec D_{m} \Pi^{j)}, B_{0 n} \succ \approx 0 \\
& C_{i}^{\prime}=<F_{i j}, \Pi^{j}>-<P^{j}, D_{i} B_{0 j}>+<A_{i}, \Upsilon>\approx 0 \\
& C_{0}^{\prime} \approx 0
\end{aligned}
$$

By a direct computation it is easy to check that $\kappa_{0}, \kappa_{i}, \Upsilon^{(\varepsilon) a}, C_{0}^{\prime}, C_{i}^{\prime}$ are first class constraints. The remaining constraints turn out to be second class. To show that this is indeed the case, we compute their Dirac matrix (i.e., the matrix of the Poisson brackets of the second class constraints)and prove its invertibility.

It appears that all the Poisson brackets of the remaining constraints are weakly ultralocal except $\left\{T^{i j}(x), T^{k l}(y)\right\}$ which contains non ultralocal term of $\delta^{\prime}(x, y)$ 
type. This can be taken care of (leading to simpler brackets) by redefining $T^{i j}$ as follows:

$$
\tilde{T}^{i j}(x)=T^{i j}(x)+\int d y X_{(k l)}^{(i j)}(x, y) \Psi(\Pi, \Pi)^{k l}(y)
$$

where $X_{(k l)}^{(i j)}(x, y)=-\frac{1}{2}\left\{T^{i j}(x), T^{k^{\prime} l^{\prime}}(y)\right\} G_{k^{\prime} l^{\prime} k l}^{-1}(y)$, so that all the Poisson brackets are now weakly ultralocal (i.e., contain only $\delta(x, y)$ and none of its derivatives). Thus, if we denote by $\chi_{\alpha}$ the set of redefined second class contraints, we now have

$$
\left\{\chi_{\alpha}(x), \chi_{\beta}(y)\right\}=\Delta_{\alpha \beta}(x) \delta(x, y) .
$$

We shall exhibit the coefficients $\Delta_{\alpha \beta}$.

The Dirac matrix has the following structure:

\begin{tabular}{|c||c|c|c|c|c|c|}
\hline$\Delta_{\alpha^{\prime} \alpha}$ & $\Phi(B, B)_{m n}$ & $\Phi(\Pi, P)^{r s}$ & $\Phi(B, \Pi)_{v}^{u}$ & $\Phi(B, P)_{i}^{j}$ & $\Phi(\Pi, \Pi)^{p q}$ & $T^{k l}$ \\
\hline \hline$\Phi(B, B)_{m^{\prime} n^{\prime}}$ & 0 & $A_{m^{\prime} n^{\prime}}^{r s}$ & 0 & 0 & 0 & 0 \\
\hline$\Phi(\Pi, P)^{r^{\prime} s^{\prime}}$ & $-A_{m n}^{r^{\prime} s^{\prime}}$ & 0 & 0 & $K_{i}^{j r^{\prime} s^{\prime}}$ & 0 & $M^{r^{\prime} s^{\prime} k l}$ \\
\hline$\Phi(B, \Pi)_{v^{\prime}}^{u^{\prime}}$ & 0 & 0 & 0 & $B_{v^{\prime} i}^{u^{\prime} j}$ & 0 & $N_{v^{\prime}}^{u^{\prime} k l}$ \\
\hline$\Phi(B, P)_{i^{\prime}}^{j^{\prime}}$ & 0 & $-K_{i^{\prime}}^{j^{\prime} r s}$ & $-B_{i^{\prime} v}^{j^{\prime} u}$ & 0 & 0 & $Q_{i^{\prime} k l}^{j^{\prime}}$ \\
\hline$\Phi(\Pi, \Pi)^{p^{\prime} q^{\prime}}$ & 0 & 0 & 0 & 0 & 0 & $G^{p^{\prime} q^{\prime} k l}$ \\
\hline$\tilde{T}^{k^{\prime} l^{\prime}}$ & 0 & $-M^{r s k^{\prime} l^{\prime}}$ & $-N_{v}^{u k^{\prime} l^{\prime}}$ & $-Q_{i}^{j k^{\prime} l^{\prime}}$ & $-G^{p q k^{\prime} l^{\prime}}$ & 0 \\
\hline
\end{tabular}

where the tensor $A, B, K, M, N, Q$ can be easily written down. In order to compute the determinant, only the values of $A$ and $B$ are necessary (besides the already known $G$ ) and we shall thus give explicitly here only those. We have:

$$
\begin{aligned}
& A_{m^{\prime} n^{\prime}}^{r s}(x)=\frac{2}{3} \mathcal{V} \delta_{\left(m^{\prime}\right.}^{(r} \delta_{\left.n^{\prime}\right)}^{s)} \\
& B_{v^{\prime} i}^{u^{\prime} j}(x)=\frac{1}{3} \mathcal{V}\left(\delta_{v^{\prime}}^{j} \delta_{i}^{u^{\prime}}-\frac{1}{3} \delta_{i}^{j} \delta_{v^{\prime}}^{u^{\prime}}\right)
\end{aligned}
$$

Proposition 1 The determinant of the Dirac matrix is:

$$
D_{2}=\operatorname{det} \Delta \approx \operatorname{det}(A)^{2} \operatorname{det}(B)^{2} \operatorname{det}(G)^{2}=2^{8} 3^{-28} \mathcal{V}^{40} V_{s}^{8}
$$

Proof: A developpement of the determinant of the Dirac Matrix along lines and columns give immediately the first equality. The second equality is obtained by pluging the explicit expression of $\operatorname{det}(A), \operatorname{det}(B), \operatorname{det}(G)$ which are given by:

$$
\operatorname{det}(A)=\left(\frac{2}{3}\right)^{6} \mathcal{V}^{6}, \operatorname{det}(B)=\left(\frac{1}{3}\right)^{8} \mathcal{V}^{8}
$$

Because this determinant does not vanish, the constraints $\chi_{\alpha}$ are indeed all second class. 


\section{III.2.2. Dirac brackets}

Due to the simple structure of the Dirac matrix, the expressions of the cofactors are easy to obtain explicitly and appear to be polynomials in the dynamical variables and their derivatives. This implies that we can compute exactly the inverse of the Dirac matrix and we will denote by $\{,\}_{D}$ the Dirac bracket. We will not give the explicit expression of the Dirac bracket of the dynamical variables in the present work because no interesting structure seems to show up. In particular, we have seen no immediate hint for a quantum group structure.

\section{III.2.3. Counting degrees of freedom}

The counting of the degrees of freedom is direct: there are 72 dynamical variables $\left(A_{i}, \Pi^{i}, B_{0 i}, P^{i}\right), 40$ second class constraints and 14 first class constraints. Therefore, the reduced phase space is 4 dimensional, which is correctly the number of degrees of freedom of general relativity.

The 14 first class constraints $\kappa_{0}, \kappa_{i}, C_{0}^{\prime}, C_{i}^{\prime}, \Upsilon^{(\varepsilon) a}$ are related to the gauge symmetries of the theory. As was pointed out above, the constraint functions $\Upsilon^{(\varepsilon) a}$ generate infinitesimal gauge transformations of type $\mathcal{G}$. As to the constraints $\kappa_{0}, \kappa_{i}, C_{0}^{\prime}, C_{i}^{\prime}$, they are related to the spacetime diffeomorphisms. The primary constraints $\kappa_{0}=0, \kappa_{i}=0$ appear because we treat the components $B_{0 i}$, which contain the lapse $N$ and the shift $N^{i}$, as dynamical variables (so, the constraints $\kappa_{0}=0, \kappa_{i}=0$ are equivalent to the constraints $\pi_{N}=0, \pi_{N^{i}}=0$ ). The secondary constraints $C_{0}^{\prime}=0, C_{i}^{\prime}=0$ are equivalent to the Hamiltonian and momentum constraints.

Note that as a consequence of the constraints, the Hamiltonian $H$ weakly vanishes. This was somehow to be expected, since the theory is diffeomorphisminvariant.

\section{Path Integral Measure}

This section aims at defining the path integral of the Plebanski theory restricted to the gravitational sector. Many difficulties have to be overcome:

1. to formulate a formal path integral taking into account the second class constraints

2. to apply a BRST procedure to gauge fix the first class constraints

3. to regularize and/or to discretize these path integrals

4. to "renormalize" these amplitudes

Our (modest) contribution will cover only the first point. We will define the path integral measure in term of the Liouville measure on the phase space taking into account the second class constraints. We will then apply the general method 
of [16] to rewrite this path integral measure as a path integral on the original configuration variables of the Lagrangian formalism.

We first give a brief introduction to the path integral method for pure secondclass constrained systems [17, 18, 10, which is rather direct. We start with a hamiltonian system with $2 n$ coordinates $\left(x^{I}\right)_{I}$ on a symplectic phase space $(\mathcal{P}, \omega)$ and a set of $2 m$ regular and irreducible second class constraints $\left(\chi_{\alpha}\right)_{\alpha}$. The reduced phase space $\left(\mathcal{C}, \omega_{D}\right)$ is the submanifold $\mathcal{C}=\left\{p \in \mathcal{P} \mid \chi_{\alpha}(p)=0, \forall \alpha=\right.$ $1, \cdots, 2 m\}$ endowed with the Dirac symplectic structure $\omega_{D}$.

The path integral is formally defined as a sum over all paths satisfying the constraints and the integral measure is given by the Liouville measure associated to $\omega_{D}$. To be more precise, let us introduce a complete set $y^{i}$ of $2(n-m)$ independent coordinates on $\mathcal{C}$ and we obtain the following definition of the path integral:

$$
\mathcal{Z} \equiv \int\left[\mathcal{D} y^{i}(t)\right] \prod_{t \in\left[t_{1}, t_{2}\right]} \sqrt{\operatorname{det} \omega_{D}(y(t))} \exp (i S[y(t)])
$$

In the previous formula, $S[y(t)]$ is the action of the path $y(t)$ and $\operatorname{det} \omega_{D}$ is the determinant of the symplectic structure.

For our purpose, it will be useful to rewrite the path integral in terms of the original variables $\left(x^{I}\right)_{I}$. To that end, we perform a coordinate transformation of the non-reduced phase space $\mathcal{P}$ from the coordinates $\left(x^{I}\right)_{I}$ to the coordinates $\left(y^{i}, \chi_{\alpha}\right)_{i, \alpha}$. To go further, we use the fact that it is always possible to find coordinates $\left(y^{i}\right)_{i}$ of the submanifold $\mathcal{C}$ such that $\left\{y^{i}, \chi_{\alpha}\right\}=0$. The local symplectic structure of $\mathcal{P}$ is therefore given by the tensor product $\omega=\omega_{D} \oplus \omega_{\chi}$ where $\left(\omega_{\chi}^{\alpha \beta}\right)_{\alpha, \beta}$ is the inverse of the Dirac matrix $\Delta_{\alpha \beta}=\left\{\chi_{\alpha}, \chi_{\beta}\right\}$ and defines the two form $\omega_{\chi}=\left(\omega_{\chi}^{\alpha \beta}\right) d \chi_{\alpha} \wedge d \chi_{\beta}$.

As a consequence, the equation relating the volume elements in each coordinates system is obtained using the Liouville measure conservation under coordinates transformations:

$$
\sqrt{\operatorname{det} \omega} \prod_{I} d x^{I}=\frac{\sqrt{\operatorname{det} \omega_{D}}}{\sqrt{\operatorname{det} \Delta}} \prod_{\alpha} d \chi^{\alpha} \prod_{i} d y^{i} .
$$

From this result, one obtains the following expression of the path integral in term of the original variables $x^{I}$ :

$$
\begin{aligned}
\mathcal{Z}=\int[\mathcal{D} x(t)] \prod_{t}(\sqrt{\operatorname{det} \omega(x(t))} \sqrt{\operatorname{det} \Delta(x(t))}) \\
\quad \times \prod_{\alpha=1}^{2 m} \delta\left(\chi_{\alpha}(x(t))\right) \exp (i S[x(t)]) .
\end{aligned}
$$

Thus, we see that the key point in writing the path integral is the computation of the Dirac matrix and the evaluation of its determinant. This task was carried 
out above for the Plebanski theory in subsection III.2.1 and the determinant was denoted $D_{2}$.

In the case at hand, there are also first class constraints. These can be gauge fixed by using 14 gauge fixing functions $\xi_{\alpha}$ chosen such that $D_{1}=\left(\operatorname{det}\left\{\psi_{\alpha}, \xi_{\beta}\right\}_{D}\right)^{2}$ does not vanish on the phase space (we collectively denote the first class constraints by $\left.\psi_{\alpha}\right)$. Once the gauge is fixed, there are only second class constraints left and one can apply the above formula. This yields (for the Plebanski theory restricted to the gravitational sector)

$$
\begin{aligned}
& \mathcal{Z}_{P l}=\int\left[\mathcal{D} A_{i}\right]\left[\mathcal{D} \Pi^{i}\right]\left[\mathcal{D} B_{0 i}\right]\left[\mathcal{D} P^{i}\right] \\
& \exp \left(i \int d t \int_{\Sigma} d^{3} x\left(<\Pi^{i}, \partial_{0} A_{i}>+<P^{i}, \partial_{0} B_{0 i}>\right)\right) \\
& \prod_{x \in M} \sqrt{D_{1}} \delta\left(\psi_{\alpha}\right) \delta\left(\xi_{\alpha}\right) \prod_{x \in M} \sqrt{D_{2}} \delta(\Phi(B, P)) \\
& \times \delta(\Phi(\Pi, P)) \delta(\Phi(B, B)) \delta(\Phi(B, \Pi)) \delta(\Phi(\Pi, \Pi)) \delta(\tilde{T})
\end{aligned}
$$

Recall that the Hamiltonian vanishes when the constraints are taken into account. The gauge fixing conditions $\xi_{\alpha}$ depend on the canonical variables and can also involve explicitly the time ("time dependent canonical gauge conditions"). For the subsequent discussion, it will be simpler to take gauge conditions that do not involve the potential $A_{i}$. This is equivalent to taking gauge conditions that involve the tetrads only (and their derivatives), but not the connection. The boundary conditions to be imposed on the paths that are summed over in the path integral should be dealt with along the lines of [19].

We may re-express (71) as

$$
\begin{aligned}
& \mathcal{Z}_{P l}=\int\left[\mathcal{D} A_{i}\right]\left[\mathcal{D} \Pi^{i}\right]\left[\mathcal{D} B_{0 i}\right]\left[\mathcal{D} P^{i}\right] \\
& \exp \left(i \int d t \int_{\Sigma} d^{3} x\left(<\Pi^{i}, \partial_{0} A_{i}>+<P^{i}, \partial_{0} B_{0 i}>-\tilde{H}\right)\right) \\
& \prod_{x \in M} \sqrt{D_{1}} \delta\left(\psi_{\alpha}\right) \delta\left(\xi_{\alpha}\right) \prod_{x \in M} \sqrt{D_{2}} \delta(\Phi(B, P)) \\
& \times \delta(\Phi(\Pi, P)) \delta(\Phi(B, B)) \delta(\Phi(B, \Pi)) \delta(\Phi(\Pi, \Pi)) \delta(\tilde{T})
\end{aligned}
$$

since $\tilde{H}$, defined as

$$
-\tilde{H}=\epsilon^{m n p}<B_{0 p}, F_{m n}>+\Lambda \prec B_{0 p}, \Pi^{p} \succ,
$$

vanishes on the constraint surface.

Our aim is to rewrite this expression as a path integral over the original covariant configuration variables with the original Plebanski action. To proceed, 
we first integrate over the momenta $P^{i}$. This can be done by using the following change of variables:

$$
\left[\mathcal{D} P^{i}\right]=J[\mathcal{D} \Phi(B, P)][\mathcal{D} \Phi(\Pi, P)]\left[\mathcal{D} \kappa_{0}\right]\left[\mathcal{D} \kappa_{i}\right]
$$

where $J$ is the Jacobian of the linear transformation $P^{i} \mapsto\left(\Phi(B, P), \Phi(\Pi, P), \kappa_{0}, \kappa_{i}\right)$, easily shown to be equal to $J=\left(\prec B_{0 k}, \Pi^{k} \succ\right)^{-9}=\mathcal{V}^{-9}$. As a result we obtain:

$$
\begin{aligned}
& \mathcal{Z}_{P l}=\int\left[\mathcal{D} A_{i}\right]\left[\mathcal{D} \Pi^{i}\right]\left[\mathcal{D} B_{0 i}\right] \\
& \exp \left(i \int d t \int_{\Sigma} d^{3} x\left(<\Pi^{i}, \partial_{0} A_{i}>-\tilde{H}\right)\right) \\
& \prod_{x \in M} \sqrt{D_{1\left(P_{i}=0\right)}} \delta\left(C_{0}^{\prime}\right) \delta\left(C_{i}^{\prime}\right) \delta(\Upsilon) \delta\left(\xi_{\alpha}\right) \\
& \times \sqrt{D_{2}} \mathcal{V}^{-9} \delta(\Phi(B, B)) \delta(\Phi(B, \Pi)) \delta(\Phi(\Pi, \Pi)) \delta(\tilde{T}) .
\end{aligned}
$$

We now apply the method of [16 to eliminate the secondary second class constraints $T^{i j}$. We denote $\zeta_{1}^{i j}=-\frac{1}{4} \Phi(\Pi, \Pi)^{i j}$ as well as $\zeta_{2}^{i j}=T^{i j}$. The constraints $\zeta_{2}^{i j}$ are the secondary constraints associated with $\zeta_{1}^{i j}$ in the sense that we have $\left\{\zeta_{1}^{i j}, \tilde{H}\right\}=\zeta_{2}^{i j}$. Introducing multipliers $\lambda_{i j}$ and $\rho_{i j}$ to enforce the constraints, we can rewrite (75) as:

$$
\begin{aligned}
& \mathcal{Z}_{P l}=\int\left[\mathcal{D} A_{i}\right]\left[\mathcal{D} \Pi^{i}\right]\left[\mathcal{D} B_{0 i}\right]\left[\mathcal{D} \lambda_{i j}\right]\left[\mathcal{D} \rho_{i j}\right] \\
& \exp \left(i \int d t \int_{\Sigma} d^{3} x\left(<\Pi^{i}, \partial_{0} A_{i}>-\tilde{H}-\lambda_{i j} \zeta_{1}^{i j}-\rho_{i j} \zeta_{2}^{i j}\right)\right) \\
& \prod_{x \in M} \sqrt{D_{1\left(P_{i}=0\right)}} \delta\left(C_{0}^{\prime}\right) \delta\left(C_{i}^{\prime}\right) \delta(\Upsilon) \delta\left(\xi_{\alpha}\right) \sqrt{D_{2}} \mathcal{V}^{-9} \delta(\Phi(B, B)) \delta(\Phi(B, \Pi))
\end{aligned}
$$

We consider a canonical transformation generated by the function $F=-\rho_{i j} \zeta_{1}^{i j}$. Under this transformation the kinetic term $<\Pi^{i}, \partial_{0} A_{i}>$ is left invariant ${ }^{6}$, as well as the path integral measure and also $\lambda_{i j} \zeta_{1}^{i j}$, whereas $\tilde{H}$ is turned into

$$
\tilde{H}^{\prime}=\exp (\{F, .\})(\tilde{H})=\tilde{H}-\rho_{i j} \zeta_{2}^{i j}-2 \rho_{i j} \rho_{k l} G^{i j k l}
$$

and $\zeta_{2}^{i j}$ is turned into

$$
\zeta_{2}^{\prime i j}=\zeta_{2}^{i j}+4 \rho_{k l} G^{i j k l}
$$

\footnotetext{
${ }^{6}$ The canonical transformation depends explicitly on the parameter $\rho_{i j}$, so the kinetic term transforms with the explicit time derivative of the generator, $\delta<\Pi^{i}, \partial_{0} A_{i}>\sim \dot{\rho}_{i j} \zeta_{1}^{i j}$. Since the constraint $\zeta_{1}^{i j}$ remains invariant (and enforced through the integration over $\lambda_{i j}$ ), the kinetic term is indeed invariant on the constraint surface. Alternatively, its variation in the non-reduced phase space can be absorbed through a redefinition of $\lambda_{i j}$.
} 
(to obtain the latter results, we have used $\left\{\zeta_{1}^{i j}, \zeta_{2}^{k l}\right\}=-4 G^{i j k l}$ and $\left\{G^{i j k l}, F\right\}=0$ ). Finally, since only $A_{i}$ transforms under the canonical transformation generated by $F$, the product of the $\delta$-distributions in the path integral is invariant (we assume that the gauge conditions do not involve $A_{i}$ ). As a result, we obtain

$$
\begin{aligned}
& \mathcal{Z}_{P l}=\int\left[\mathcal{D} A_{i}\right]\left[\mathcal{D} \Pi^{i}\right]\left[\mathcal{D} B_{0 i}\right]\left[\mathcal{D} \rho_{i j}\right] \\
& \exp \left(i \int d t \int_{\Sigma} d^{3} x\left(<\Pi^{i}, \partial_{0} A_{i}>-\tilde{H}-2 \rho_{i j} \rho_{k l} G^{i j k l}\right)\right) \\
& \prod_{x \in M} \sqrt{D_{1\left(P_{i}=0\right)}} \delta\left(C_{0}^{\prime}\right) \delta\left(C_{i}^{\prime}\right) \delta(\Upsilon) \delta\left(\xi_{\alpha}\right) \sqrt{D_{2}} \mathcal{V}^{-9} \delta(\Phi(B, B)) \delta(\Phi(B, \Pi)) \delta(\Phi(\Pi, \Pi)) .
\end{aligned}
$$

The integration over the variables $\rho_{i j}$ is gaussian and gives

$$
\begin{aligned}
& \mathcal{Z}_{P l}=\int\left[\mathcal{D} A_{i}\right]\left[\mathcal{D} \Pi^{i}\right]\left[\mathcal{D} B_{0 i}\right] \exp \left(i \int d t \int_{\Sigma} d^{3} x\left(<\Pi^{i}, \partial_{0} A_{i}>-\tilde{H}\right)\right) \\
& \prod_{x \in M} \sqrt{D_{1\left(P_{i}=0\right)}} \delta\left(C_{0}^{\prime}\right) \delta\left(C_{i}^{\prime}\right) \delta(\Upsilon) \delta\left(\xi_{\alpha}\right) \frac{\sqrt{D_{2}} \mathcal{V}^{-9}}{\sqrt{\operatorname{det}(G)}} \delta(\Phi(B, B)) \delta(\Phi(B, \Pi)) \delta(\Phi(\Pi, \Pi)) .
\end{aligned}
$$

We have thus succeeded in eliminating the constraints $T_{i j}$.

To make contact with the Plebanski action, we now reintroduce the original configuration variables. Because $\Pi^{i}=\epsilon^{i j k} B_{j k}$, the Jacobian of the transformation from $\Pi^{i}$ to $B_{j k}$ is a constant, therefore $\left[\mathcal{D} \Pi^{k}\right]=\left[\mathcal{D} B_{i j}\right]$. We can furthermore eliminate the Dirac distributions $\delta(\Upsilon), \delta(\Phi(B, B)), \delta(\Phi(B, \Pi)), \delta(\Phi(\Pi, \Pi))$ by restoring the integration over $A_{0}$ and $\varphi^{\mu \nu \rho \sigma}$. Taking into account the known values of $\sqrt{D_{2}}$ and $\sqrt{\operatorname{det}(G)}$, we obtain the final expression:

$$
\begin{gathered}
\mathcal{Z}_{P l}=\int\left[\mathcal{D} A_{\mu}\right]\left[\mathcal{D} B_{\mu \nu}\right]\left[\mathcal{D} \varphi^{\mu \nu \rho \sigma}\right] \mathcal{V}^{8} V_{s}^{2} \exp \left(i S_{P l}[A, B, \varphi]\right) \\
\prod_{x \in M} \sqrt{D_{1\left(P_{i}=0\right)}} \delta\left(C_{0}^{\prime}\right) \delta\left(C_{i}^{\prime}\right) \delta\left(\xi_{\alpha}\right)
\end{gathered}
$$

This is the formal expression of the gauge fixed measure of Plebanski theory in the non topological sector. One can be puzzled by the fact that the measure has an expression which is non covariant because it contains $V_{s}^{2}$. This phenomenon is typical of derivative couplings and is completely analogous to the non covariant measure computed 40 years ago by H.Leutwyler [20] which reads:

$$
\mathcal{D} g=\prod_{x} \mathcal{V}^{-5} V_{s}^{2} \prod_{\mu \leq \nu} d g_{\mu \nu}
$$

A derivation of this measure from the Liouville measure was done by E.S.Fradkin and G.A.Vilkovisky 21, 22, as well as a careful analysis of the covariance of the correlation functions. See also [23] for the different comparisons of functional measure for quantum gravity. 


\section{Conclusion}

In the present work we have derived the hamiltonian analysis of the Plebanski theory. A notable feature of this theory is that it defines a non-regular theory, in the sense that the matrix of the Poisson brackets of the constraints does not have constant rank on the constraint surface. Non-regular theories used to be thought of as being somewhat pathological and of no interest to physical systems. In the last years, however, models have been constructed that lead to non-regular constrained Hamiltonian systems. Another example, besides Plebanski theory, is given by the higher-dimensional pure Chern-Simons theories [24, 25]. A recent analysis of non-regular systems may be found in [26].

We have explicitly carried out the derivation of the Hamiltonian formalism in the non topological sector. In particular, we have obtained all the constraints and been able to disentangle them into first class and second class. We have also computed exactly the local measure, which has enabled us to give a formal path integral of Plebanski theory (in the non topological sector) in term of the original variables. This constitutes a first step in the direction of the understanding of Plebanski theory.

Our work should be further extended in various directions. First, the above path integral was written in a canonical gauge. It would be useful to derive it in propagating gauges by BRST methods.

Second, it would also be of interest to try to discretize the Plebanski theory using spin foam models. A central issue is the computation of the weights of the faces and edges which are still a matter of debate [27, 28, 29]. The computation of the functional measure that we provide will hopefully give new insights in this intricate problem.

Finally, one of our motivations was to try to uncover a quantum group structure underlying the Plebanski theory. In euclidean BF theory with cosmological constant it is well known that quantum groups are hidden in this topological field theory [30]. Up to now there is no clear picture of appearance of quantum groups (more precisely of Poisson Lie groups or Poisson Lie groupoids) as symmetries of Poisson brackets of the fields in this theory. Unfortunately, the form of the Dirac brackets that we can compute in Plebanski theory does not appear to be connected with such structures in any obvious way. The relation, if any, between quantum groups and Plebanski theory still remains to us a mystery as well as the

relation between the quantum deformation of the Barrett-Crane model [31] and a discretization of Lorentzian Plebanski theory with cosmological constant. 


\section{Acknowledgments}

M.H. is grateful to the "Laboratoire de physique mathématique et théorique" of the University of Montpellier for kind hospitality while this work was carried out. The research of M.H. is supported in part by the "Actions de Recherche Concertées" of the "Direction de la Recherche Scientifique - Communauté Française de Belgique", by a "Pôle d'Attraction Interuniversitaire" (Belgium) and by IISNBelgium (convention 4.4505.86). Support from the European Commission RTN programme HPRN-CT-00131, in which M.H. is associated to K. U. Leuven, is also acknowledged.

The research of K.N. is supported by NSF grants PHY-0090091 and the Eberly Research Funds of Penn State University. 


\section{Appendix}

\section{Algebra $s o(\eta)$ : definition, notations and properties}

$g=s o(\eta)$ is the real Lie algebra of the isometry group $G=S O(\eta)$ of the quadratic form $\eta=\operatorname{diag}\left(\sigma^{2}, 1,1,1\right)$ where $\sigma \in\{1, i\}$. We will use capital latin letters for internal vector indices $I, J, \cdots \in\{0,1,2,3\}$ and we will define the antisymmetic tensor $\epsilon^{I J K L}$ such that $\epsilon^{0123}=1$; the indices are lowered and raised with the metric $\eta$.

One of the basis of $s o(\eta)$, composed of rotation generators $\left(J_{a}\right)_{a=1,2,3}$ and boost generators $\left(K_{a}\right)_{a=1,2,3}$ has the following commutation relations:

$$
\left[J_{a}, J_{b}\right]=\epsilon_{a b}^{c} J_{c},\left[K_{a}, K_{b}\right]=\sigma^{2} \epsilon_{a b}^{c} J_{c},\left[K_{a}, J_{b}\right]=\epsilon_{a b}^{c} K_{c} .
$$

We have used the notation $\epsilon_{a b c}=\epsilon^{0}{ }_{a b c}$.

The real Lie algebra $s o(\eta)$ is also a real form of $s o(4, \mathbb{C})=s o(3, \mathbb{C}) \oplus s o(3, \mathbb{C})$.

It is convenient to introduce the basis $\left(T_{( \pm) a}\right)_{a=1,2,3}$ defined from the previous one by $T_{( \pm) a}=\frac{1}{2}\left(J_{a} \pm \sigma K_{a}\right)$ whose generators realize two commuting copies of so(3), i.e.:

$$
\left[T_{( \pm) a}, T_{( \pm) b}\right]=\epsilon_{a b}^{c} T_{( \pm) c} \quad, \quad\left[T_{(+) a}, T_{(-) b}\right]=0 .
$$

We denote by $p^{+}$and $p^{-}$the projectors associated to this direct sum. To any element $\xi$ of $s o(4, \mathbb{C})$, we denote $\xi^{ \pm}=p^{ \pm}(\xi)=\xi^{( \pm) a} T_{( \pm) a}$.

The star structure on $s o(4, \mathbb{C})$ selecting the real form reads:

$$
\star\left(T_{( \pm) a}\right)=-T_{\left(\sigma^{2} \pm\right) a} .
$$

As usual it is customary to define the Hodge linear map $\iota=\frac{1}{\sigma}\left(p^{+}-p^{-}\right)$which satisfies $\iota: s o(\eta) \rightarrow s o(\eta)$ and $\iota^{2}=\sigma^{2} i d$, as well as:

$$
\forall \xi, \chi \in s o(\eta), \quad[\iota(\xi), \chi]=[\xi, \iota(\chi)] .
$$

The space of real symmetric invariant bilinear forms on the Lie algebra $s o(\eta)$ is a two-dimensional vector space; we choose two independent nondegenerate bilinear forms $<\cdot, \cdot>$ and $\prec \cdot, \cdot \succ$ defined by:

$$
<T_{(\varepsilon) a}, T_{\left(\varepsilon^{\prime}\right) b}>=\delta_{a b} \delta_{\varepsilon \varepsilon^{\prime}} \quad, \quad \prec T_{(\varepsilon) a}, T_{\left(\varepsilon^{\prime}\right) b} \succ=\sigma \varepsilon \delta_{a b} \delta_{\varepsilon \varepsilon^{\prime}} .
$$

From the definition of $\iota$, we have:

$$
\forall \xi, \chi \in s o(\eta), \quad \prec \iota(\xi), \chi \succ=\prec \xi, \iota(\chi) \succ=<\xi, \chi>.
$$

The vector representation $\Pi$ of the Lie algebra $s o(\eta)$ is given by:

$$
\Pi\left(T_{( \pm) a}\right)^{I J}=T_{( \pm) a}^{I J}=\frac{1}{2}\left(-\epsilon^{0 a I J} \pm \sigma\left(\eta^{a I} \eta^{0 J}-\eta^{0 I} \eta^{a J}\right)\right) .
$$


To prove that the formulae (84) define a representation of the Lie algebra, it is sufficient to verify the following useful relations:

$$
T_{(\varepsilon) a}^{I J} \eta_{J J} T_{\left(\varepsilon^{\prime}\right) b}^{J K}=\delta_{\varepsilon \varepsilon^{\prime}}\left(\frac{1}{2} \epsilon_{a b}^{c} T_{(\varepsilon) c}^{I K}-\frac{1}{4} \eta_{a b} \delta^{I K}\right)
$$

Furthermore, the values of the quadratic Casimir tensors of $s o(\eta)$ in the vector representation can be computed using the relation

$$
\sum_{a} T_{(\varepsilon) a}^{I J} T_{(\varepsilon) a}^{K L}=\frac{1}{4} \varepsilon \sigma \epsilon^{I J K L}+\frac{1}{4}\left(\delta^{I K} \delta^{J L}-\delta^{I L} \delta^{J K}\right) .
$$

From these quadratic formulae, it is easy to compute the components $\xi^{( \pm) a}$ of any element $\xi$ in the Lie algebra in term of $\xi^{I J}$ and one obtains:

$$
\xi^{( \pm) a}=-\eta^{a b} \xi^{I J} \eta_{I K} \eta_{K L} T_{( \pm) b}^{K L} .
$$

\section{References}

[1] J.F. Plebanski, "On the separation of Einstein substructures", Jour. Math. Phys. 18, 2511 (1977).

[2] R. Capovilla, T. Jacobson, J. Dell, Selfdual two forms and gravity, Class.Quant.Grav.8:41-57, 1991

[3] T.Jacobson, J. D. Romano, Degenerate Extensions of general relativity, Class.Quant.Grav.9:L119-L124,1992 arXiv:gr-qc/9207005.

[4] J.C. Baez, "An Introduction to Spin-Foam models of BF Theory and Quantum Gravity", Lect. Notes Phys. 543, 25-94, (2000) arXiv:gr-qc/9905087.

[5] A. Perez, "Spin Foam models for quantum gravity", Class. Quant. Grav. 20, R43, (2003) arXiv:gr-qc/0301113.

[6] G. Horowitz, "Exactly Soluble Diffeomorphism Invariant Theories", Comm. Math. Phys, 125 417-437, (1989).

[7] D. Birmingham, M. Blau, M. Rakowski and G. Thompson, Phys. Rept. 209, 129 (1991).

[8] A.S. Cattaneo, P. Cotta-Ramusino, J. Fröhlich, M. Martellini, "Topological BF theories in 3 and 4 dimensions", Jour. Math. Phys. 36, 6137-6160, (1995) arXiv:hep-th/9505027. 
[9] E. Witten, "2+1 dimensional gravity as an exactly soluble system”, Nucl. Phys. B 311, 46-78, (1988).

[10] M. Henneaux, C. Telteiboim, "Quantization of gauge systems", Princeton University Press, New Jersey, (1992).

[11] M. Mondragon, M. Montesinos, "Covariant canonical formalism for 4dimensional BF theory," arXiv:gr-qc/0402041.

[12] L. Freidel, K. Krasnov, R. Puzio, "BF description of higher-dimensional gravity theories ", Adv. Theor. Math. Phys. 3, 1289-1324, (1999), arXiv:hep-th/9901069.

[13] M. Reisenberger, "Classical Euclidean general relativity from 'left-handed area=right-handed area" ", arXiv:gr-qc/9804071.

[14] R. De Pietri, L. Freidel, "SO(4) Plebanski action and relativistic Spin-Foam model", Class. Quant. Grav. 16, 2187-2196, (1999) arXiv:gr-qc/9804071.

[15] H. Urbantke, "On integrability properties of SU(2) Yang-Mills fields. I.Infinitesimal part", J. Math. Phys. 25, 2321 (1984).

[16] M. Henneaux, A. Slavnov, "A note on the path integral for systems with primary and secondary second class constraints", Phys. Lett. B 338, 47-50, (1994) arXiv:hep-th/9406161.

[17] P. Senjanovic, "Path Integral Quantization Of Field Theories With Second Class Constraints," Annals Phys. 100 (1976) 227 [Erratum-ibid. 209 (1991) 248].

[18] L. D. Faddeev, "Feynman Integral For Singular Lagrangians," Theor. Math. Phys. 1, 1 (1969) [Teor. Mat. Fiz. 1, 3 (1969)].

[19] M. Henneaux, C. Teitelboim and J. D. Vergara, Nucl. Phys. B 387, 391 (1992) arXiv:hep-th/9205092.

[20] H. Leutwyler, "Gravitational Field: Equivalence of Feynman Quantization and Canonical quantization," Phys.Rev, Vol 134, Number 5B, B1156, (1964).

[21] E.S. Fradkin and G.A. Vilkovisky, "S Matrix for Gravitational Field: II. Local measure; General relations; Elements of Renormalization theory.", Phys. Rev. D, Volume 8, Number 12, 4241 (1973).

[22] E. S. Fradkin and G. A. Vilkovisky, "Quantization Of Relativistic Systems With Constraints: Equivalence Of Canonical And Covariant Formalisms In Quantum Theory Of Gravitational Field," CERN-TH-2332 
[23] M. Kaku and P. Senjanovic, "Functional measure for quantum gravity", Phys. Rev. D, Volume 15, Number 14, 1019 (1977).

[24] M. Banados, L. J. Garay and M. Henneaux, "The Local degrees of freedom of higher dimensional pure Chern-Simons theories," Phys. Rev. D 53, 593 (1996) arXiv:hep-th/9506187.

[25] M. Banados, L. J. Garay and M. Henneaux, "The dynamical structure of higher dimensional Chern-Simons theory," Nucl. Phys. B 476, 611 (1996) arXiv:hep-th/9605159.

[26] O. Miskovic and J. Zanelli, "Dynamical structure of irregular constrained systems," J. Math. Phys. 44, 3876 (2003) arXiv:hep-th/0302033.

[27] J. Baez,J. Christensen,T. Halford and D. Tsang, " Spin Foam Models of Riemannian Quantum Gravity," Class.Quant.Grav. 19 4627-4648 (2002)[arXiv: gr-qc/0202017].

[28] L. Freidel,D. Louapre, "Diffeomorphisms and spin foam models", Nucl.Phys. B662 (2003) 279-298, [arXiv: gr-qc/0212001] .

[29] M. Bojowald and A. Perez, "Spin Foam Quantization and Anomalies," arXiv:gr-qc/0303026.

[30] L. Crane and D. Yetter, "A categorical construction of 4D topological quantum field theory", Quantum Topology, (Ser.Knots Everything Vol 3) (River edges, NJ:World Scientific) pp 120-130.

[31] K. Noui and Ph. Roche, "Cosmological deformation of Lorentzian Spin Foam Models", Class.Quant.Grav. 20 3175-3214 (2003). [arXiv: gr-qc/0211109] 